\title{
Analysis of Behaviour Pattern in Sprague-Dawley Rats Using Microwave Techniques
}

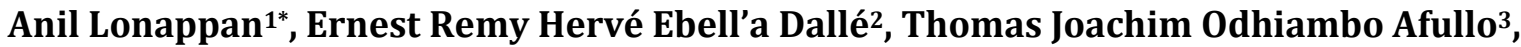 Williams Mark Uren Daniels ${ }^{2}$}

\author{
${ }^{1}$ Department of Electrical Engineering, Mangosuthu University of Technology, Durban, South Africa \\ ${ }^{2}$ College of Health Sciences, School of Laboratory Medicine and Medical Sciences, University of KwaZulu-Natal, Durban, \\ South Africa \\ ${ }^{3}$ School of Electrical Electronic and Computer Engineering, Howard College, University of KwaZulu-Natal, Durban, South Africa \\ Email: *lonappan@mut.ac.za
}

How to cite this paper: Lonappan, A., Dallé, E.R.H.E., Afullo, T.J.O. and Daniels, W.M.U. (2017) Analysis of Behaviour Pattern in Sprague-Dawley Rats Using Microwave Techniques. Journal of Electromagnetic Analysis and Applications, 9, 155-166. https://doi.org/10.4236/jemaa.2017.911014

Received: August 16, 2017

Accepted: November 20, 2017

Published: November 23, 2017

Copyright $\odot 2017$ by authors and Scientific Research Publishing Inc.

This work is licensed under the Creative

Commons Attribution International

License (CC BY 4.0). http://creativecommons.org/licenses/by/4.0/

\begin{abstract}
This communication presents a new method of detecting the behaviour pattern in Sprague-Dawley (SD) rats based on the measurement of the dielectric properties of blood plasma at microwave frequencies at different periods of time. The microwave measurements were performed by rectangular cavity perturbation method in the S-band of microwave frequency with the blood plasma collected from normal rats (Controls) as well as chemically induced rats $(\mathrm{A} \beta)$. A change is observed in the dielectric properties of the $\mathrm{A} \beta$ samples but not the controls samples at the extended period of time. This measurement technique is simple and the collection of blood from the rats is nonsurgical in nature. These results prove a new method of diagnosing Alzheimer's Disease $(\mathrm{AD})$ using microwave techniques.
\end{abstract}

\section{Keywords}

Blood Plasma, Sprague-Dawley Rats, Cavity Perturbation, Alzheimer's Disease

\section{Introduction}

Alzheimer's Disease, (AD) is the most common form of dementia with no cure at the moment. It worsens as it progresses, and eventually leads to death. The concept of senile dementia has evolved from a rather vague notion that mental decline occurred inevitably in old age, to become defined today by a distinct set of clinical and pathological features with the potential for treatment and preven- 
tion within grasp [1]. Alzheimer's Disease (AD) is diagnosed in people over 65 years of age although the less-prevalent early-onset $\mathrm{AD}$ can occur much earlier. Modern advances in therapeutic and preventive strategies that lead to even small delays in the onset and progression of Alzheimer's Disease can significantly reduce the global burden of this disease [2] [3].

The microwave technology is often used in various diagnostic and therapeutic applications in medicine [4]. Dielectric properties of various human tissues and body fluids are studied and reported at different RF frequencies [5] [6] [7]. Various measurement methods are used to measure the complex permittivity of a material and the method of choice depends on factors like the nature of the samples under study and the frequency range used [8] [9] [10] [11]. When only very small volumes of the sample are available, the cavity perturbation technique is an attractive option as it requires only minute volumes for the measurement [12]. This makes it suitable for the dielectric study of blood plasma, as only very small volumes can be extracted from the Sprague-Dawley rats. However, no data is currently available for the complex permittivity of blood plasma of Sprague-Dawley rats in the literature. In this study the rectangular cavity perturbation technique has been employed for the measurement of the dielectric properties of blood plasma obtained from normal rats (Controls) as well as chemically induced rats ( $\mathrm{A} \beta$ samples) in the frequency range 2 to $4 \mathrm{GHz}$. It is observed that an appreciable a change in the dielectric properties of $\mathrm{A} \beta$ samples than the controls samples at the extended period of time. This microwave measurement procedure as well as the extraction of blood plasma from Sprague-Dawley rats is simple and nonsurgical in nature. These results prove a new method of diagnosing Alzheimer's Disease using microwave diagnostic procedures.

\section{Sample Preparation}

The study was conducted on Sprague-Dawley (SD) rats, weighing between 300 $350 \mathrm{~g}$ (8/9 weeks of age). The animals were randomly divided into 4 groups upon arrival and housed with two animals/cage under standard lighting conditions of 12 hour light/dark cycles of protocol [13]. They were given 7 days to acclimatize to the new environment before the experiments were conducted. They were given ad libitum and bedding was changed at least two times a week.

Pre-lesion behavioral trials were conducted using Morris Water Maze (MWM) in the temperature range $23^{\circ} \mathrm{C}-30^{\circ} \mathrm{C}$ and in order to assess the learning capacities of the animals from day 1 to 3 . On day 5 , each animal was given atropine $(0.1 \mathrm{mg} / \mathrm{kg}$, i.p.) and subsequently anaesthetized using sodium pentobarbital (60 mg/kg, i.p.). After full anaesthesia (the absence of hind paw reflex), the animals stereotaxically received an intra-cerebral injection of $10 \mu \mathrm{l}(5 \mu \mathrm{l} / \mathrm{per}$ site bilateral lesions) of Amyloid $\beta$-protein (A $\beta_{42}$ dissolved in Tris buffer solution of $0.15 \mathrm{M}$ to reach a concentration of $10 \mu \mathrm{g}$ ) directly into the CA1 region of the left and right dorsal hippocampi. The animals were given a 2 days recovery period following intra-cerebral injections. The animals then repeated the behavior- 
al in the same MWM to assess any impairment in the recall ability caused by the neurotoxin. The animals were sacrificed 3, 7, 10 and 14 days following intra-cerebral injections by Halothane overdose. Blood was collected by cardiac puncture into heparin blood tubes, centrifuged at $3500 \mathrm{rpm}$ for 10 minutes, and plasma was collected in eppendorfs, snap frozen by liquid nitrogen and stored at $-80^{\circ} \mathrm{C}$. The blood plasma was then filled into capillary bulb for microwave measurements.

\section{Material and Methods}

The experimental set-up consists of a transmission type S-band rectangular cavity resonator, Rohde \& Schwarz ZVL network analyser. The cavity resonator is a transmission line with one or both ends closed. The numbers of resonant frequencies are determined by the length of the resonator. The resonator in this set-up is excited in the $\mathrm{TE}_{10 \mathrm{p}}$ mode. The sample holder which is made of glass in the form of a capillary tube flared to a disk shaped bulb at the bottom is placed into the cavity through the non-radiating cavity slot, at broader side of the cavity which can facilitate the easy movement of the holder. The resonant frequency $f_{o}$ and the corresponding quality factor $Q_{o}$ of the cavity at each resonant peak with the empty sample holder placed at the maximum electric field are noted. The same holder filled with known amount of sample under study is again introduced into the cavity resonator through the non-radiating slot. The resonant frequencies of the sample loaded cavity is selected and the position of the sample is adjusted for maximum perturbation (i.e. maximum shift of resonant frequency with minimum amplitude for the peak). The new resonant frequency $f_{s}$ and the quality factor $Q_{s}$ are noted. The same procedure is repeated for other resonant frequencies. The measurement are made from $2 \mathrm{GHz}$ to $4 \mathrm{GHz}$ of S-band in microwave region [14].

\section{Theory}

When a material is introduced into a resonant cavity, the cavity field distribution and resonant frequency are changed which depend on shape, electromagnetic properties and its position in the fields of the cavity. Dielectric material interacts only with electric field in the cavity.

According to the theory of cavity perturbation, the complex frequency shift is related as [14]:

$$
-\frac{\mathrm{d} \Omega}{\Omega} \approx \frac{\left(\overline{\varepsilon_{r}}-1\right) \int_{V_{s}} E \cdot E_{0}^{*} \mathrm{~d} V}{2 \int_{V_{c}}\left|E_{0}\right|^{2} \mathrm{~d} V}
$$

But

$$
\frac{\mathrm{d} \Omega}{\Omega} \approx \frac{\mathrm{d} \omega}{\omega}+\frac{j}{2}\left[\frac{1}{Q_{s}}-\frac{1}{Q_{0}}\right]
$$

Equating (1) and (2) and separating real and imaginary parts results 


$$
\begin{gathered}
\varepsilon_{r}^{\prime}-1=\frac{f_{o}-f_{s}}{2 f_{s}}\left(\frac{V_{c}}{V_{s}}\right) \\
\varepsilon_{r}^{\prime \prime}=\frac{V_{c}}{4 V_{s}}\left(\frac{Q_{o}-Q_{s}}{Q_{o} Q_{s}}\right)
\end{gathered}
$$

Here, $\overline{\varepsilon_{r}}=\varepsilon_{r}^{\prime}-j \varepsilon_{r}^{\prime \prime}, \overline{\varepsilon_{r}}$ is the relative complex permittivity of the sample, $\varepsilon_{r}^{\prime}$ is the real part of the relative complex permittivity, which is known as dielectric constant. $\varepsilon_{r}^{\prime \prime}$ is the imaginary part of the relative complex permittivity associated with the dielectric loss of the material. $V_{s}$ and $V_{c}$ are corresponding volumes of the sample and the cavity resonator. The conductivity can be related to the imaginary part of the complex dielectric constant as

$$
\sigma_{e}=\omega \varepsilon^{\prime \prime}=2 \pi f \varepsilon_{0} \varepsilon_{r}^{\prime \prime}
$$

\section{Results and Discussion}

The microwave experiment in blood plasma was done using cavity perturbation technique collected from normal rats (Controls) as well as chemically induced rats (A $\beta$ samples) and the results are shown in Figures 1-16. The Figures 1-4 shows the dielectric values and the corresponding Figures 9-12 shows the average values of the dielectric constant. From Figures 1-3 it is noticed that the controls as well as $\mathrm{A} \beta$ samples exhibit similar ranges of dielectric constant. This shows that dielectric constant of controls and $\mathrm{A} \beta$ samples from day 3 , day 7 and day 10 have almost same values. This is quite evident from the average value

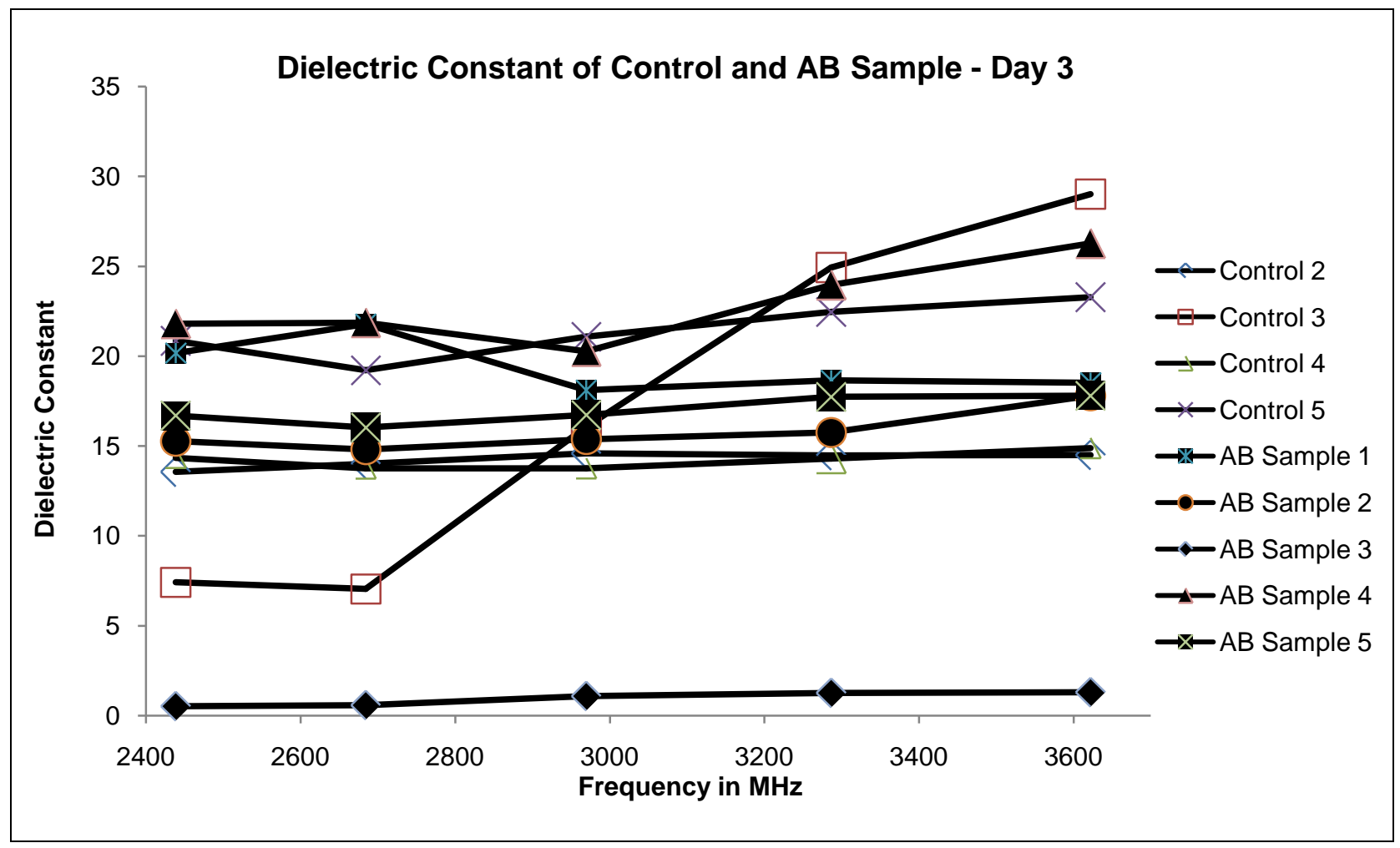

Figure 1. Variation of dielectric constant in control and $\mathrm{A} \beta$ samples after 3 days. 


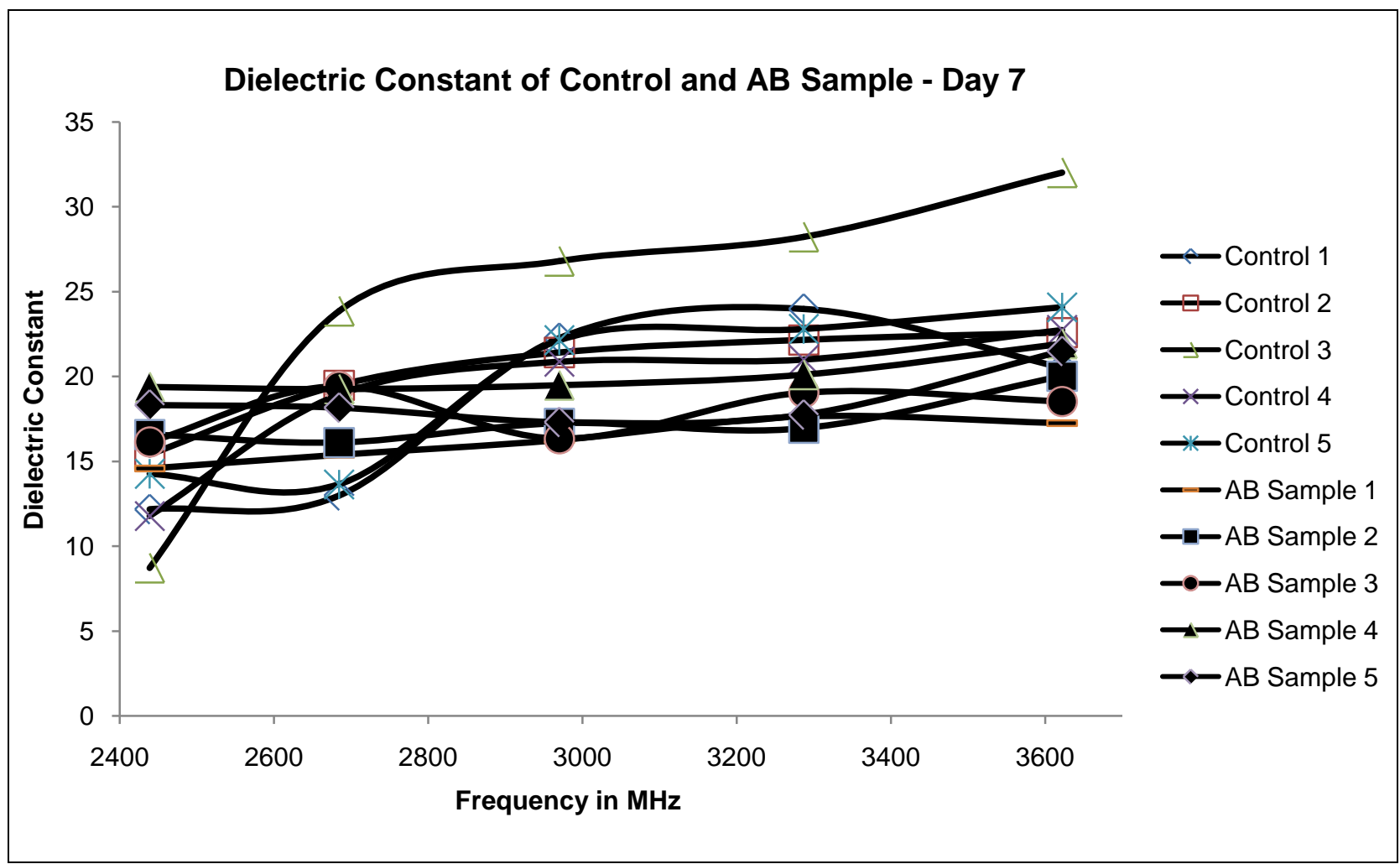

Figure 2. Variation of dielectric constant in control and $\mathrm{A} \beta$ samples after 7 days.

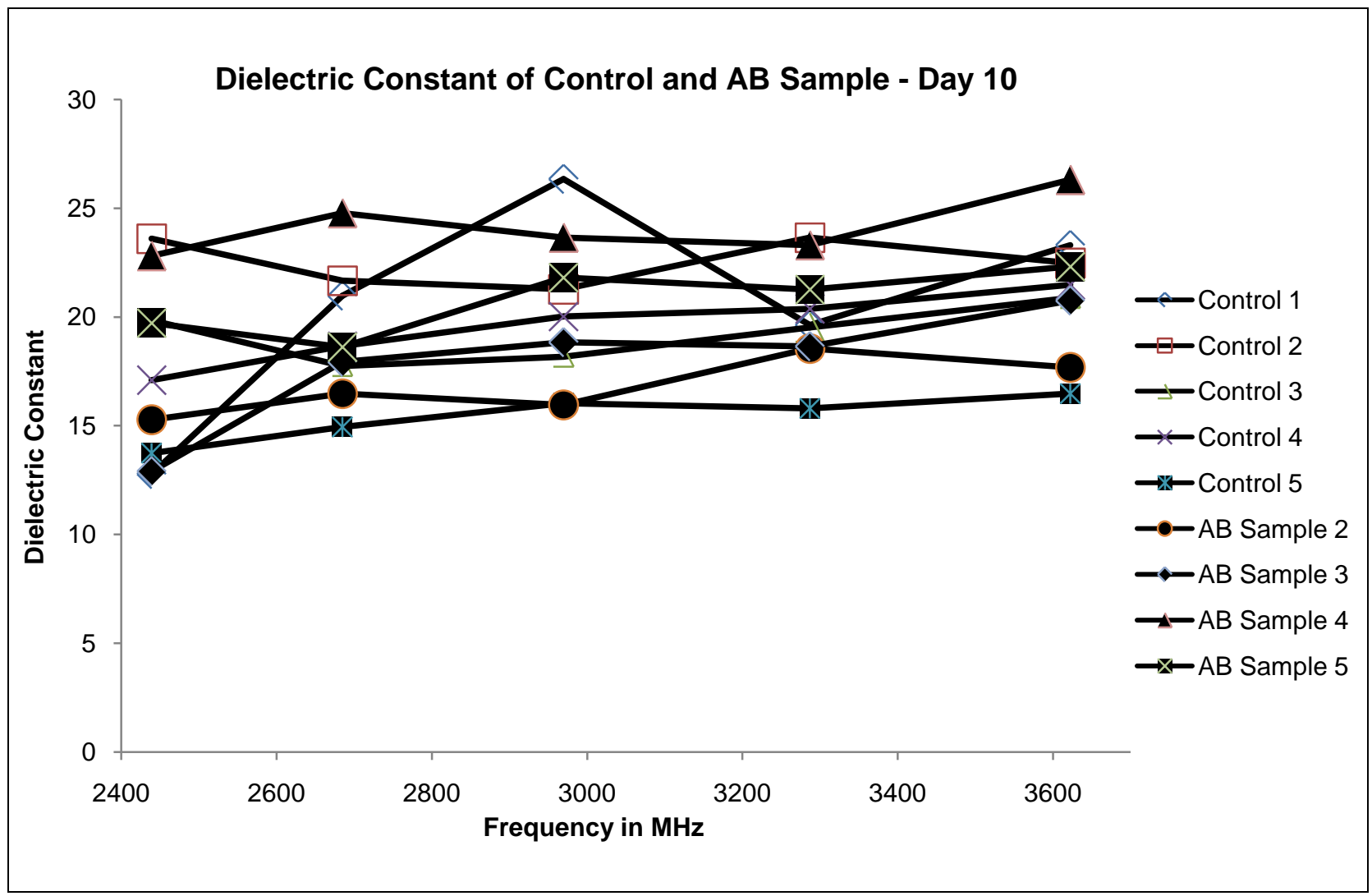

Figure 3. Variation of dielectric constant in control and $\mathrm{A} \beta$ samples after 10 days. 


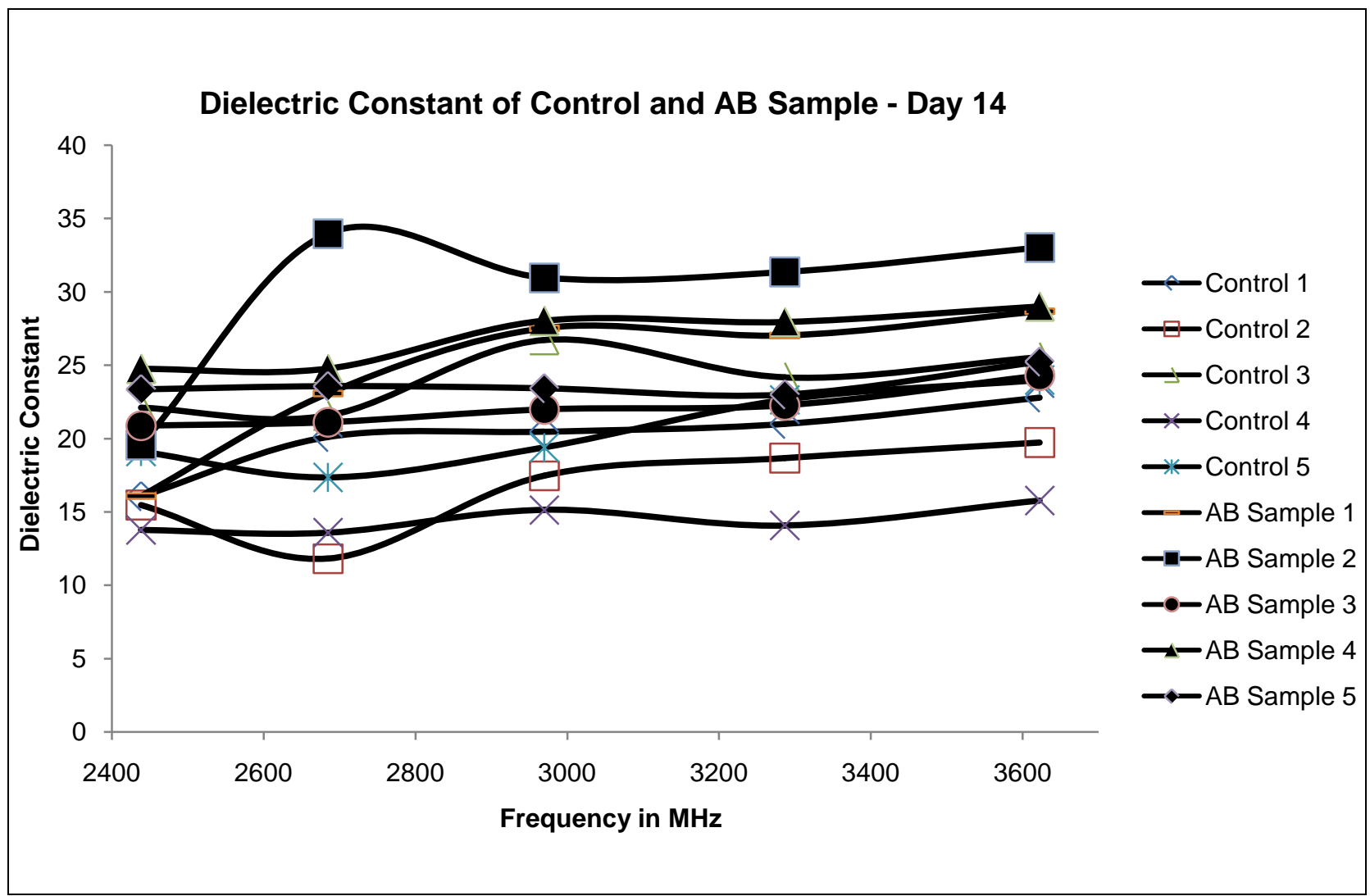

Figure 4. Variation of dielectric constant in control and $\mathrm{A} \beta$ samples after 14 days.

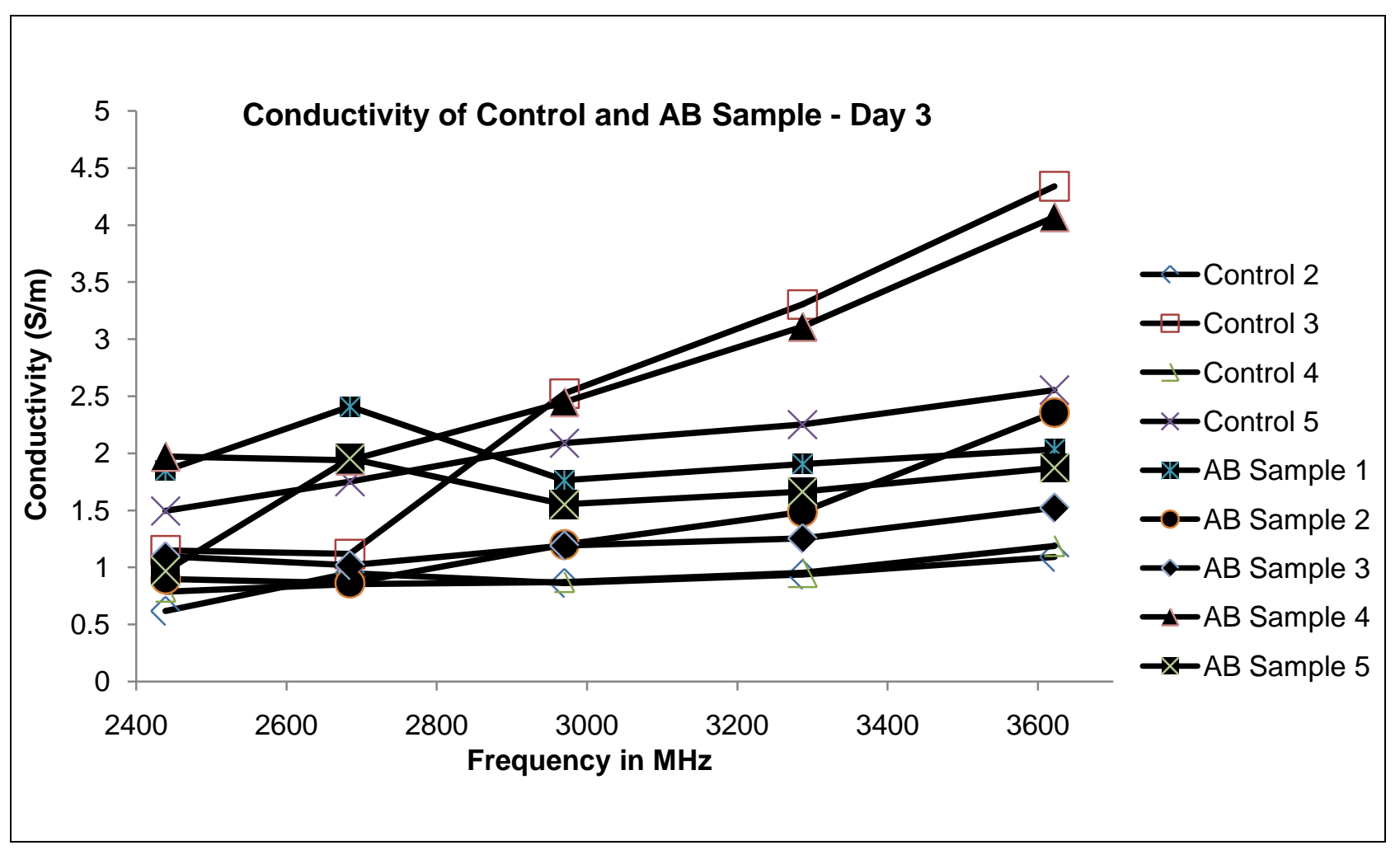

Figure 5. Variation of conductivities in control and $\mathrm{A} \beta$ samples after 3 days. 


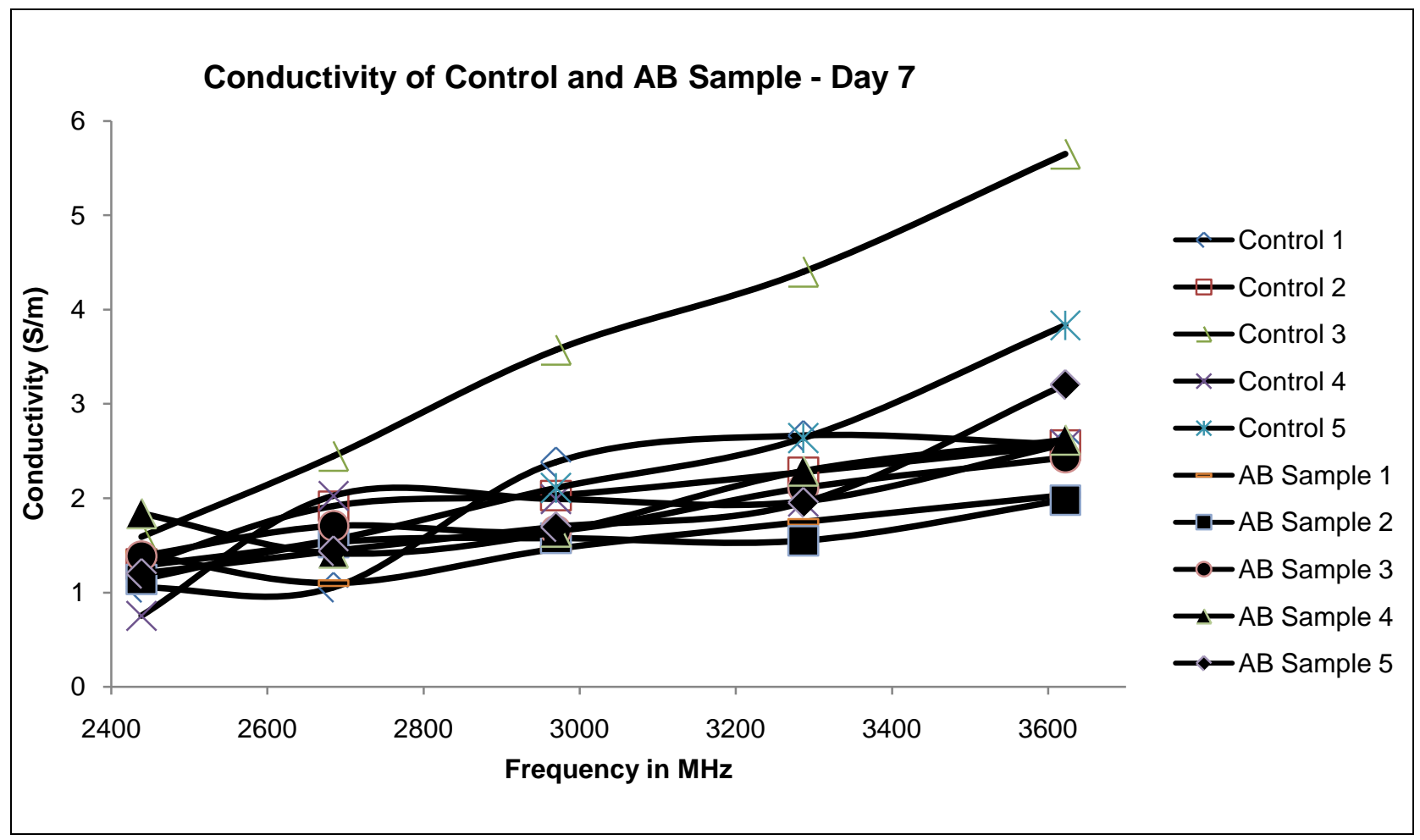

Figure 6. Variation of conductivities in control and $\mathrm{A} \beta$ samples after 7 days.

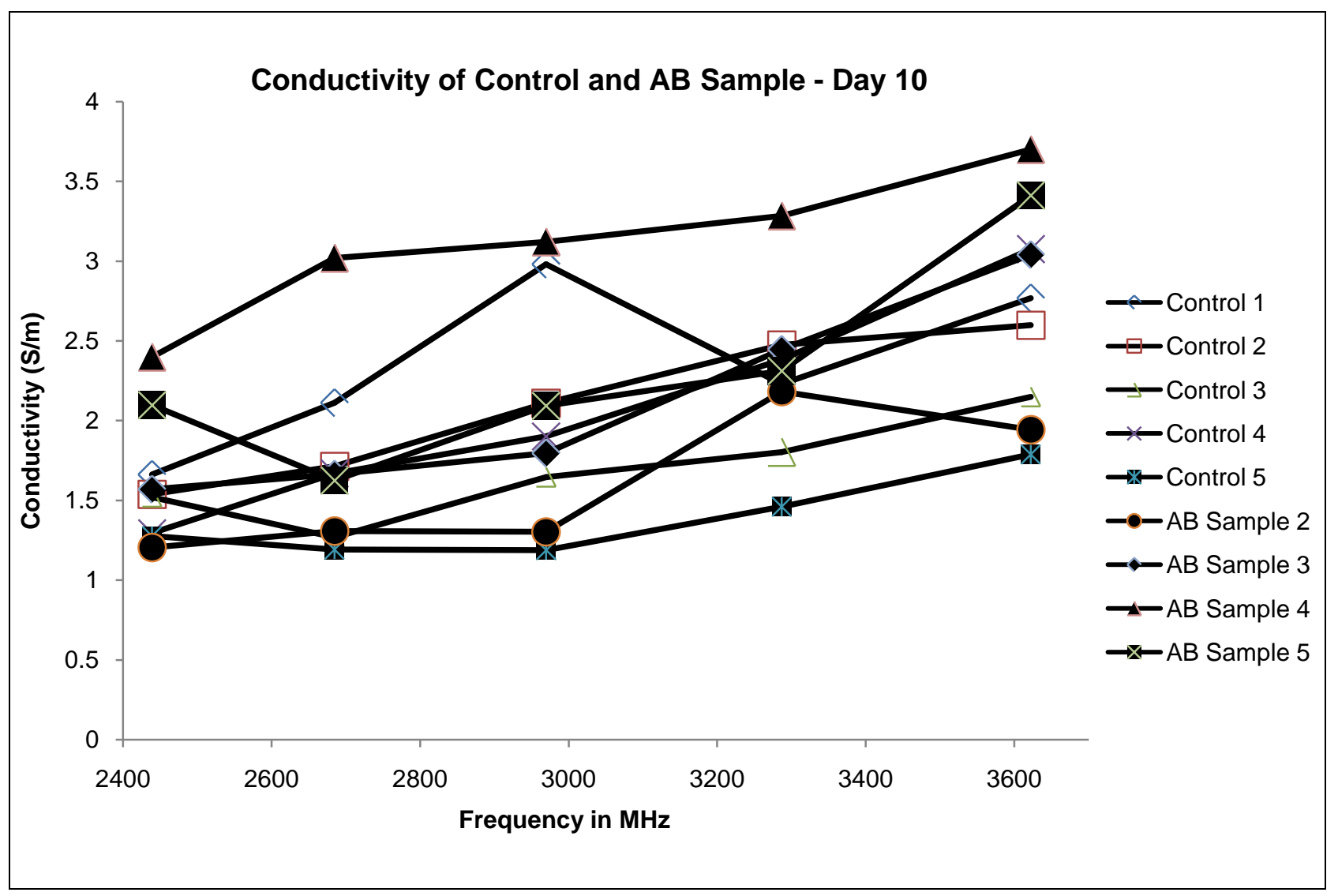

Figure 7. Variation of conductivities in control and $\mathrm{A} \beta$ samples after 10 days. 


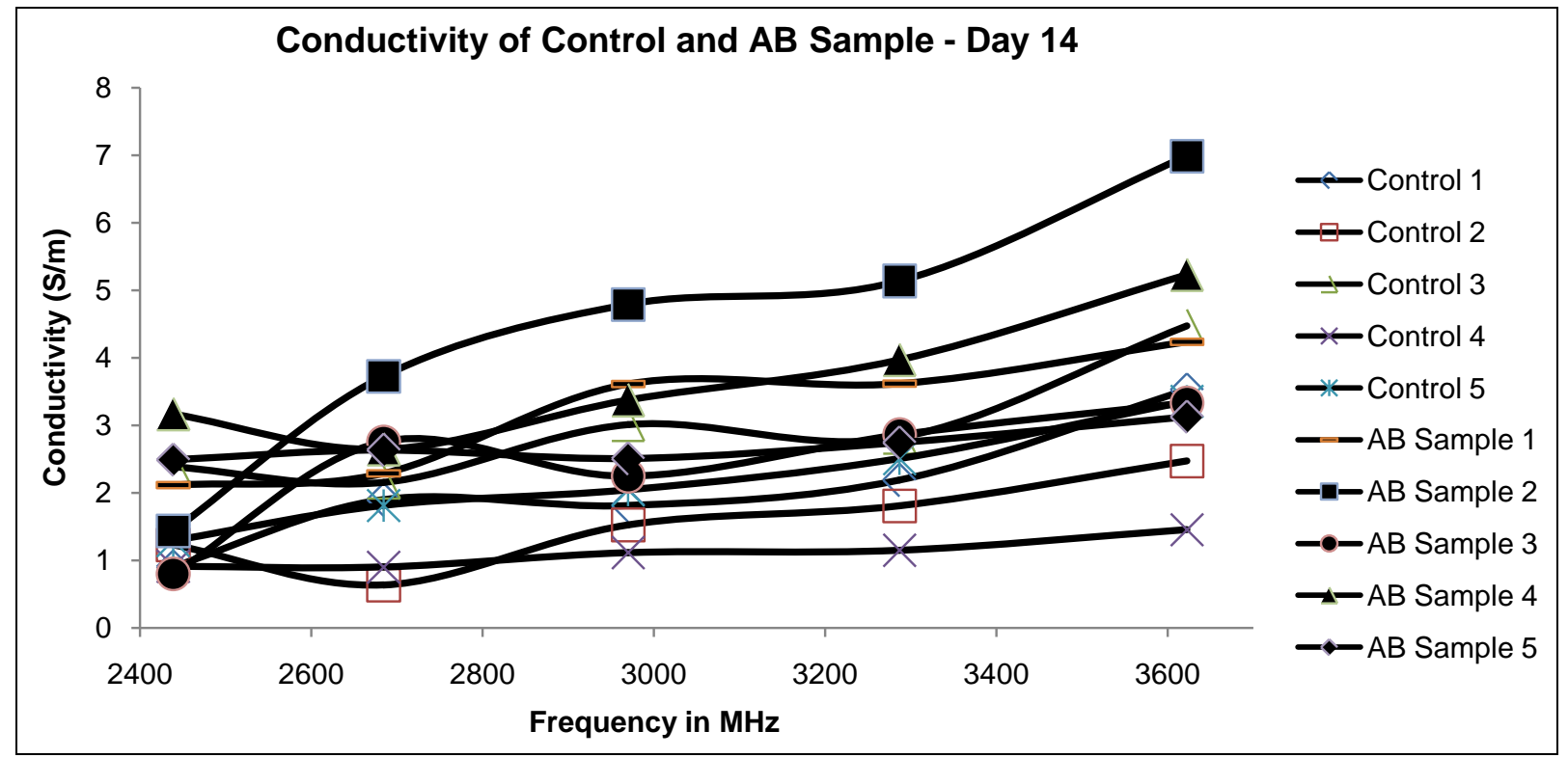

Figure 8. Variation of conductivities in control and $\mathrm{A} \beta$ samples after 14 days.

3 days dielectric constant

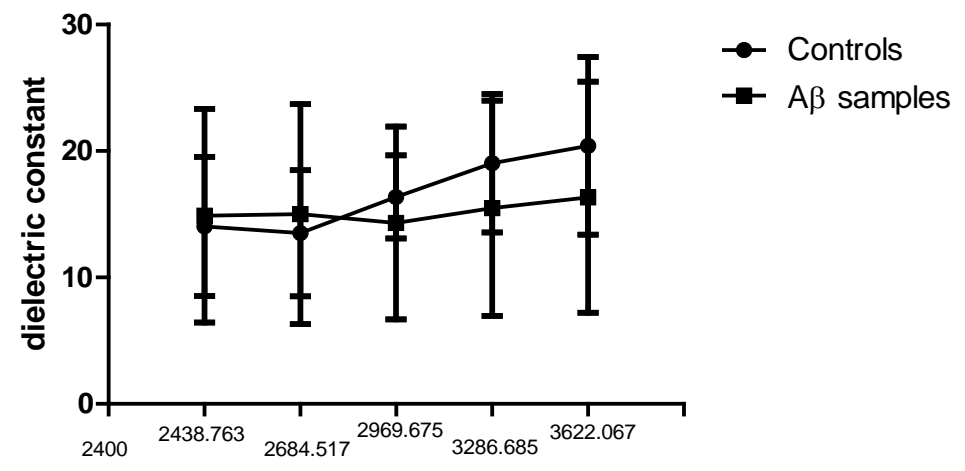

Frequency (MHz)

Figure 9. Variation of the average dielectric constant of controls and $\mathrm{A} \beta$ samples after 3-days.

7 days dielectric constant

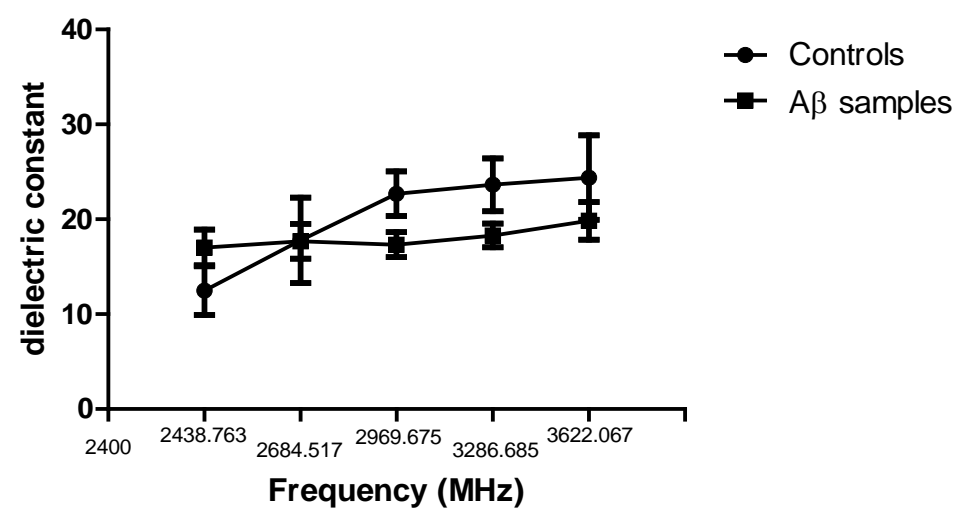

Figure 10. Variation of the average dielectric constant of controls and $\mathrm{A} \beta$ samples after 7-days. 


\section{0 days dielectric constant}

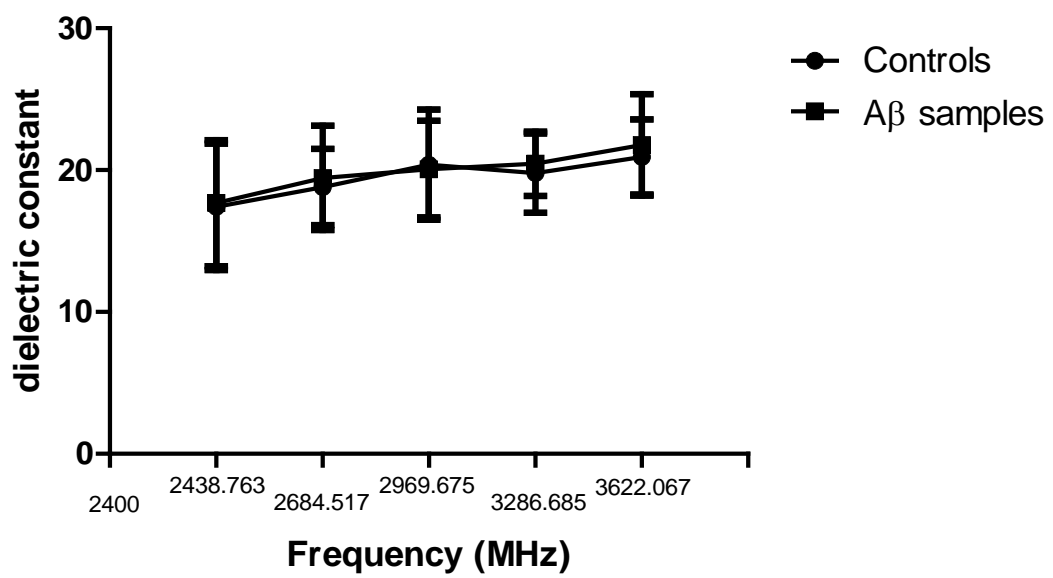

Figure 11. Variation of the average dielectric constant of controls and $\mathrm{A} \beta$ samples after 10-days.

\section{4 days dielectric constant}

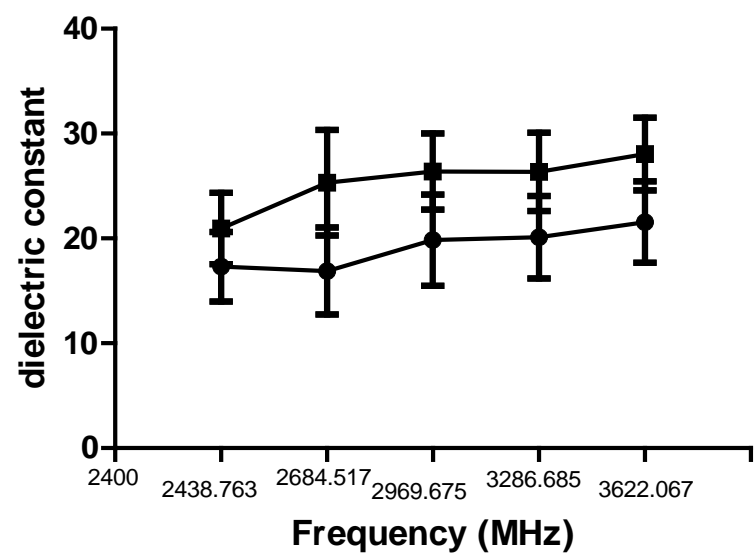

$\rightarrow$ Controls
$\rightarrow-A \beta$ samples

Figure 12. Variation of the average dielectric constant of controls and $\mathrm{A} \beta$ samples after 14-days.

results dielectric constants shown in the Figures 9-11. From Figure 4 as well as Figure 12, $\mathrm{A} \beta$ samples exhibit a higher dielectric constant than that the controls. This implies that dielectric constant in $\mathrm{A} \beta$ samples is higher than the controls after day 14 and is due to the change in the behaviour of samples attributed by Alzheimer's Disease. The Figures 5-8 shows the conductivity values and the corresponding Figures 13-16 shows the average values of the conductivities. Figure 5 to Figure 6 show the variation of conductivity of blood plasma of control and $A \beta$ samples and its values are practically similar. The average values of conductivities during same period is shown in Figure 13 and Figure 14. From Figure 7 and Figure 8, it is observed that there is a distinct variation in the conductivities of blood plasma of $\mathrm{A} \beta$ samples to controls. The corresponding average values of these period is shown in Figure 15 and Figure 16. The increase in conductivity in $\mathrm{A} \beta$ samples than the controls due to the change in the behaviour of samples attributed by Alzheimer's Disease. Thus in the S band of 


\section{3 days conductivity}

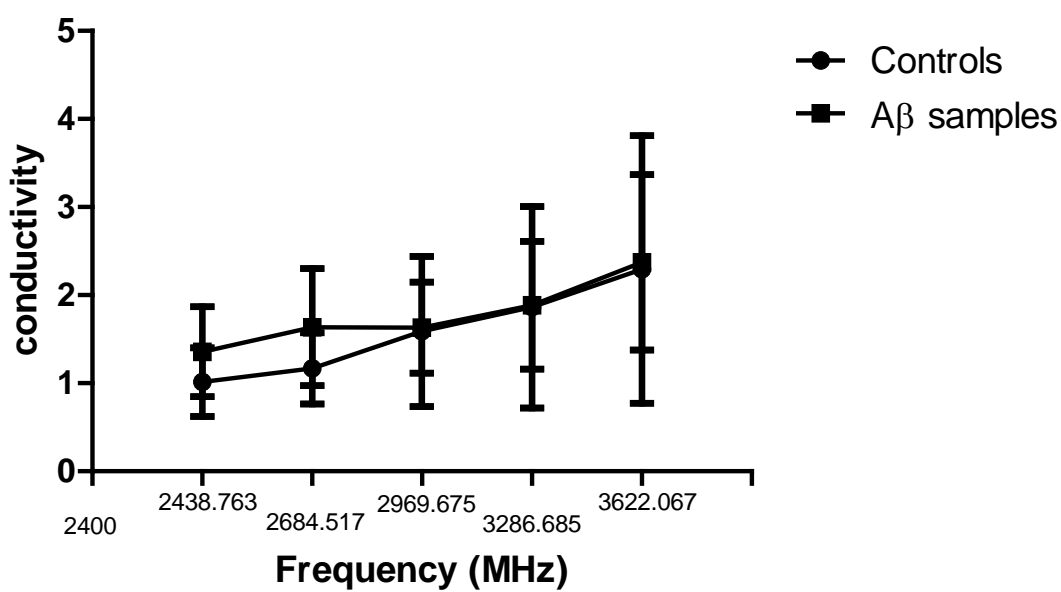

Figure 13. Variation of the average conductivity of controls and $\mathrm{A} \beta$ samples after 3-days.

\section{7 days conductivity}

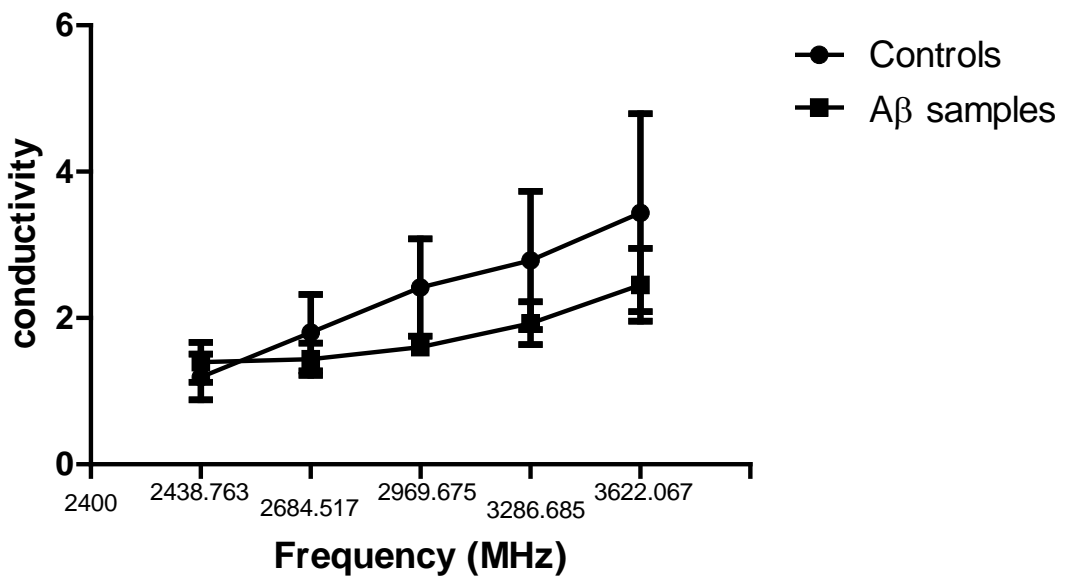

Figure 14. Variation of the average conductivity of controls and $\mathrm{A} \beta$ samples after 7 -days.

\section{0 days conductivity}

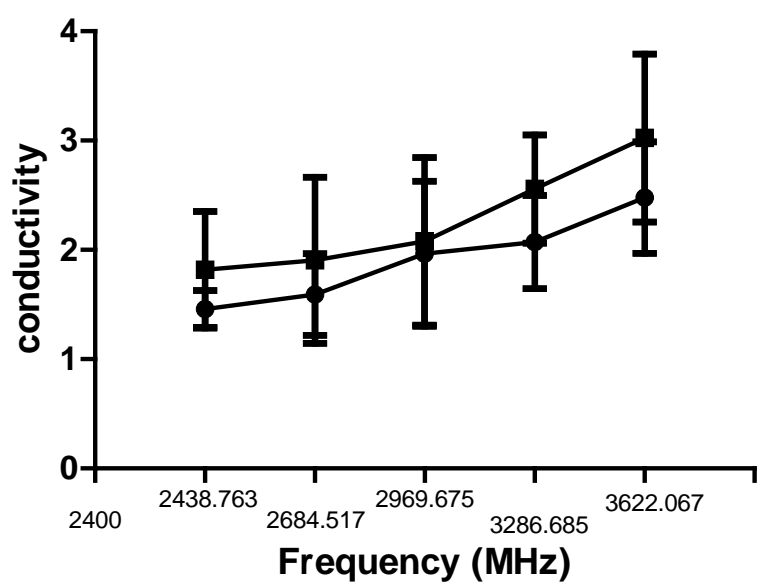

$\rightarrow$ Controls
$\rightarrow A \beta$ samples

Figure 15. Variation of the average conductivity of controls and $\mathrm{A} \beta$ samples after 10-days. 


\section{4 days conductivity}

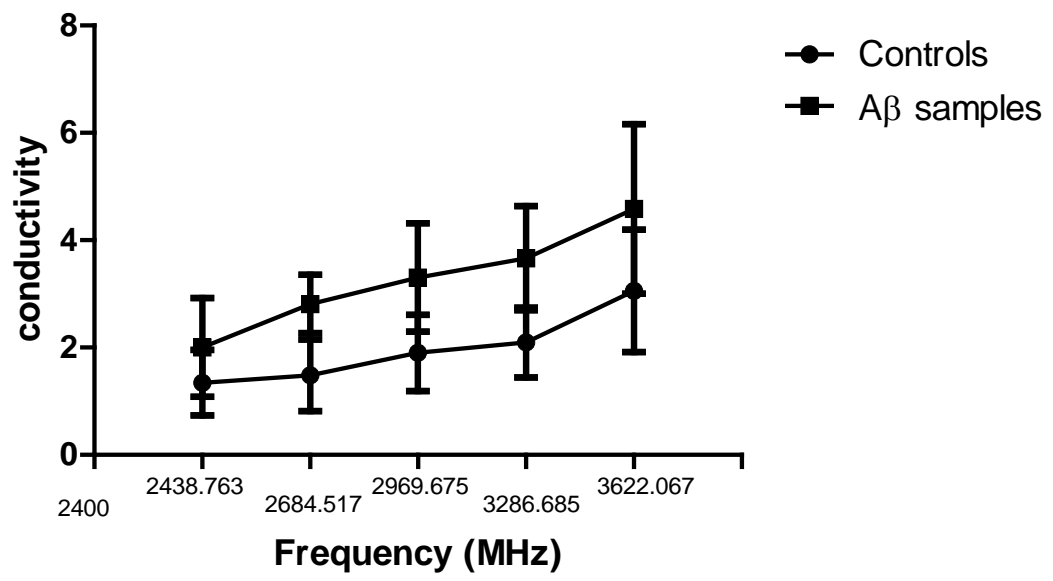

Figure 16. Variation of the average conductivity of controls and $\mathrm{A} \beta$ samples after 14-days.

microwave (ISM band), controls and A $\beta$ samples of blood plasma of Sprague-Dawley rats were studied and exhibit distinct variation of dielectric constant and conductivity after 14 days.

\section{Conclusion}

The microwave characterization has been performed in the blood plasma obtained from normal rats (Controls) as well as chemically induced rats (A $\beta$ samples) using the cavity perturbation technique. The cavity perturbation technique is quick, simple, and accurate and it requires very low volume of sample for measuring the dielectric properties of samples like blood plasma. It is observed that a substantial change in the dielectric properties of $A \beta$ samples occurs on day 14. This measurement technique as well as the extraction of blood samples from Sprague-Dawley rats is simple. These results prove a new method of diagnosing Alzheimer's Disease using microwave techniques.

\section{Acknowledgements}

This work done by Anil Lonappan is the part of his Doctoral studies in Electronic Engineering at the School of Electrical Electronic and Computer Engineering, University of KwaZulu Natal. The work done by Ernest Remy Hervé Ebell'a Dalle is the part of his Honours degree in human physiology at the School of Laboratory Medicine and Medical Sciences, University of KwaZulu-Natal.

\section{References}

[1] Berchtold, N.C. and Cotman, C.W. (1998) Evolution in the Conceptualization of Dementia and Alzheimer's Disease: Greco-Roman Period to the 1960s. Neurobiology of Aging, 19, 173-189.

[2] Brookmeyer, R., Gray, S. and Kawas, C. (1998) Projections of Alzheimer's Disease 
in the United States and the Public Health Impact of Delaying Disease Onset. American Journal of Public Health, 88, 1337-1342. https://doi.org/10.2105/AJPH.88.9.1337

[3] Brookmeyer, R., Johnson, E., Ziegler Graham, K. and Arrighi, H.M. (2007) Forecasting the Global Burden of Alzheimer's Disease. Alzheimer's \& Dementia, 3, 186-191. https://doi.org/10.1016/j.jalz.2007.04.381

[4] Rosen, A., Stuchly, M.A. and Vorst, A.V. (2002) Applications of RF/Microwaves in Medicine. IEEE Transactions on Microwave Theory and Techniques, 50.

[5] Gabriel, S., Lau, R.W. and Gabriel, C. (1996) The Dielectric Properties of Biological Tissues: II. Measurements on the Frequency Range $10 \mathrm{~Hz}$ to $20 \mathrm{GHz}$. Literature Survey. Physics Medicine Biology, 41, 2251-2269. https://doi.org/10.1088/0031-9155/41/11/002

[6] Cook, H.F. (1951) Dielectric Behavior of Human Blood at Microwave Frequencies. Nature, 168, 247-248. https://doi.org/10.1038/168247a0

[7] Cook, H.F. (1951) The Dielectric Behavior of Some Types of Human Tissues at Microwave Frequencies. British Journal of Applied Physics, 2, 295-300. https://doi.org/10.1088/0508-3443/2/10/304

[8] Ghodgaonkar, D.K., Varadan, V.V. and Varadan, V.K. (1990) Free Space Measurement of Complex Permittivity and Complex Permeability of Magnetic Materials at Microwave Frequencies. IEEE Transactions on Instrumentation and Measurement, 19, 387-394. https://doi.org/10.1109/19.52520

[9] Ghodgaonkar, D.K., Varadan, V.V. and Varadan, V.K. (1989) A Free Space Method for Measurement of Dielectric Constant and Loss Tangents at Microwave Frequencies. IEEE Transaction on Instrumentation and Measurement, 38, 789-793. https://doi.org/10.1109/19.32194

[10] Barry, W. (1986) A Broadband, Automated, Stripline Technique for the Simultaneous Measurement of Complex Permittivity and Complex Permeability. IEEE Transaction on Microwave Theory and Techniques, 34, 80-84. https://doi.org/10.1109/TMTT.1986.1133283

[11] Abbas, Z., Pollard, R.D. and Kelsall, R.W. (1998) A Rectangular Dielectric Waveguide Technique for Determination of Permittivity of Materials at W-Band. IEEE Transactions on Microwave Theory and Techniques, 46, 2011-2015. https://doi.org/10.1109/22.739275

[12] Mathew, K.T. (2005) Perturbation Theory. In: Mathew, K.T., Ed., Encyclopedia of $R F$ and Microwave Engineering, Vol. 4, Wiley-Interscience, USA, 3725-3735. https://doi.org/10.1002/0471654507.eme309

[13] Sipos, E., Kurunczi, A., Kasza, Á., Horváth, J., Felszeghy, K., Laroche, S., Toldi, J., Párducz, Á., Penke, B. and Penke, Z. (2007) $\beta$-Amyloid Pathology in the Entorhinal Cortex of Rats Induces Memory Deficits: Implications for Alzheimer's Disease. Neuroscience, 147, 28-36.

[14] Lonappan, A., Afullo, T.J.O. and Daniels, W.M.U. (2017) Analysis of Certain Fruit Juices Using Microwave Techniques. Journal of Electromagnetic Analysis and Applications, 9, 123-134. https://doi.org/10.4236/jemaa.2017.99011 